\title{
BMJ Open Long-term risk factors for suicide in suicide attempters examined at a medical emergency in patient unit: results from a 32-year follow-up study
}

\author{
Sara Probert-Lindström (10 ,1,2 Jonas Berge, ${ }^{1,3}$ Åsa Westrin, ${ }^{2,4}$ Agneta Öjehagen, ${ }^{1}$ \\ Katarina Skogman Pavulans ${ }^{1,5}$
}

To cite: Probert-Lindström S, Berge J, Westrin $\AA$, et al. Longterm risk factors for suicide in suicide attempters examined at a medical emergency in patient unit: results from a 32-year follow-up study. BMJ Open 2020;10:e038794. doi:10.1136/ bmjopen-2020-038794

\section{- Prepublication history} materials for this paper is available online. To view these files, please visit the journal online (http://dx.doi.org/10. 1136/bmjopen-2020-038794).

Received 24 March 2020 Revised 02 July 2020 Accepted 08 October 2020

Check for updates

(c) Author(s) (or their employer(s)) 2020. Re-use permitted under CC BY-NC. No commercial re-use. See rights and permissions. Published by BMJ.

${ }^{1}$ Department of Clinical Sciences, Lund University, Lund, Sweden

${ }^{2}$ Region Skåne, Clinical Psychiatric Research Center, Lund, Sweden

${ }^{3}$ Addiction Center Malmö,

Malmo, Sweden

${ }^{4}$ Division of Psychiatry, Lund University Department of Clinical Sciences Malmo, Lund, Sweden

${ }^{5}$ Child and Adolescent

Psychiatry, Karlskrona, Sweden

Correspondence to Sara Probert-Lindström; sara.probert-lindstrom@med. lu.se

\section{ABSTRACT}

Objectives The overall aim of this study is to gain greater knowledge about the risk of suicide among suicide attempters in a very long-term perspective. Specifically, to investigate possible differences in clinical risk factors at short ( $\leq 5$ years) versus long term ( $>5$ years), with the hypothesis that risk factors differ in the shorter and longer perspective.

Design Prospective study with register-based follow-up for 21-32 years.

Setting Medical emergency inpatient unit in the south of Sweden.

Participants 1044 individuals assessed by psychiatric consultation when admitted to medical inpatient care for attempted suicide during 1987-1998.

Outcome measures Suicide and all-cause mortality. Results At follow-up, $37.6 \%$ of the participants had died, $7.2 \%$ by suicide and $53 \%$ of these within 5 years of the suicide attempt. A diagnosis of psychosis at baseline represented the risk factor with the highest $\mathrm{HR}$ at longterm follow-up, that is, $>5$ years, followed by major depression and a history of attempted suicide before the index attempt. The severity of a suicide attempt as measured by SIS (Suicide Intent Scale) showed a nonproportional association with the hazard for suicide over time and was a relevant risk factor for suicide only within the first 5 years after an attempted suicide.

Conclusions The risk of suicide after a suicide attempt persists for up to 32 years after the index attempt. A baseline diagnosis of psychosis or major depression or earlier suicide attempts continued to be relevant risk factors in the very long term. The SIS score is a better predictor of suicide risk at short term, that is, within 5 years than at long term. This should be considered in the assessment of suicide risk and the implementation of care for these individuals.

\section{INTRODUCTION}

Suicide is a preventable cause of premature death and is highly associated with mental distress (for a recent review see ${ }^{1}$ ). The most well-documented risk factor for suicide is a history of attempted suicide. ${ }^{23}$ The incidence of suicide is highest within the first years of the attempt but the risk of suicide seems to persist
Strengths and limitations of this study

- The observation time of up to 32 years enabled a long-term investigation of suicide risk.

- A large clinical population $(\mathrm{n}=1044)$ provided sufficient statistical power for subgroup comparisons.

- The total number of suicides $(n=75)$ limited the number of variables that could be included in the analyses, thereby risking the omission of potentially important variables.

- Access to a broad set of clinically relevant baseline data that were collected in a standardised manner was a strength, but the reliability of psychiatric diagnoses was limited by the emergency setting and single diagnostic assessor.

- The follow-up data were limited to register data of survival and cause of death.

for many years after a suicide attempt. ${ }^{4-12}$ Long-term studies of mortality among suicide attempters have found that between $2 \%$ and $13 \%$ have died by suicide after 20-37 years after the index suicide attempt. ${ }^{10-14}$

Several studies have examined the risk factors for suicide in the first few years following a suicide attempt and have among other variables identified: male sex and older age, ${ }^{3}{ }^{15-18}$ mental disorder, ${ }^{19}$ substance abuse, ${ }^{161920}$ previous psychiatric care,${ }^{2021}$ earlier suicide attempts, ${ }^{4}{ }^{16} 21$ violent method $^{2122}$ and suicidal intent ${ }^{23}$ as relevant variables. Studies assessing long-term risk factors for suicide among suicide attempters are far more scarce. Studies with a total observation time of $10-19$ years ${ }^{57824-27}$ report mostly the same risk factors as shorter-term studies. To our knowledge, there are only three previous prospective studies (two of mainly the same sample, ${ }^{11} 14$ including uncertain suicide) of suicide risk factors among suicide attempters with follow-up periods of 20 or more years, reporting male sex, ${ }^{12}$ a baseline 
diagnosis of schizophrenia, bipolar/unipolar depressive disorder, other depression ${ }^{11}$ and an index suicide attempt involving hanging, strangulation or suffocation. ${ }^{14}$ One of these was based on a small clinical sample $(\mathrm{n}=98),{ }^{12}$ and the other two were register studies with very large samples $(\mathrm{n}=39685, \mathrm{n}=48649),{ }^{11}{ }^{14}$ but without evaluation of clinical risk factors other than the method used during the index suicide attempt and diagnosis at discharge from inpatient care following the suicide attempt.

As previous studies of mortality rates among suicide attempters point at a sustained elevated risk for suicide over many years after attempted suicide, ${ }^{4-12}$ there is a need for more knowledge on long-term risk factors and whether they differ from short-term risk factors. Only two previous studies ${ }^{827}$ involved subgroup comparisons of risk factors for those who died by suicide within the first years after the index attempt and those who committed suicide after that. Tidemalm $e t a l l^{8}$ including both certain and uncertain cases of suicide, indicated that violent method at index attempt and mental disorder increase the suicide risk within the first year in young men and that repeated self-harm may increase the long-term (2-9 years) risk in young patients. Maser $e t a l^{27}$ found that, in a sample of affectively ill patients, suicide within a year was best predicted by clinical variables such as symptoms of panic attacks, whereas suicide beyond 1 year was better predicted by personality variables such as impulsivity. None of these studies investigated risk factors at the very long term (over 20 years).

The overall aim of the present study is to investigate the occurrence of suicide and all-cause mortality, and risk factors for suicide, over an interval lasting up to 32 years after the index attempt. A further aim is to investigate the possible differences between risk factors for suicide in the proximal ( $\leq 5$ years) and the distant ( $>5$ years) intervals, with the hypothesis that different risk factors are important in a shorter and longer perspective.

Research questions:

- What proportion of the suicide attempters had died by suicide at long-term follow-up?

- What are the risk factors for suicide at long-term follow-up?

- Are the clinical factors associated with risk of suicide at short term following attempted suicide the same as those associated with risk of suicide at long term? If not, what differences can be found?

Patient and public involvement

Patients or the public were not involved in this study.

\section{METHOD}

\section{Study design and cohort collection}

The present study is a second follow-up of a population previously studied ${ }^{28}$ and consists of clinical interview and register data from a large population $(n=1044)$ of suicide attempters admitted to a medical emergency inpatient unit (MEIU), where they were thoroughly interviewed

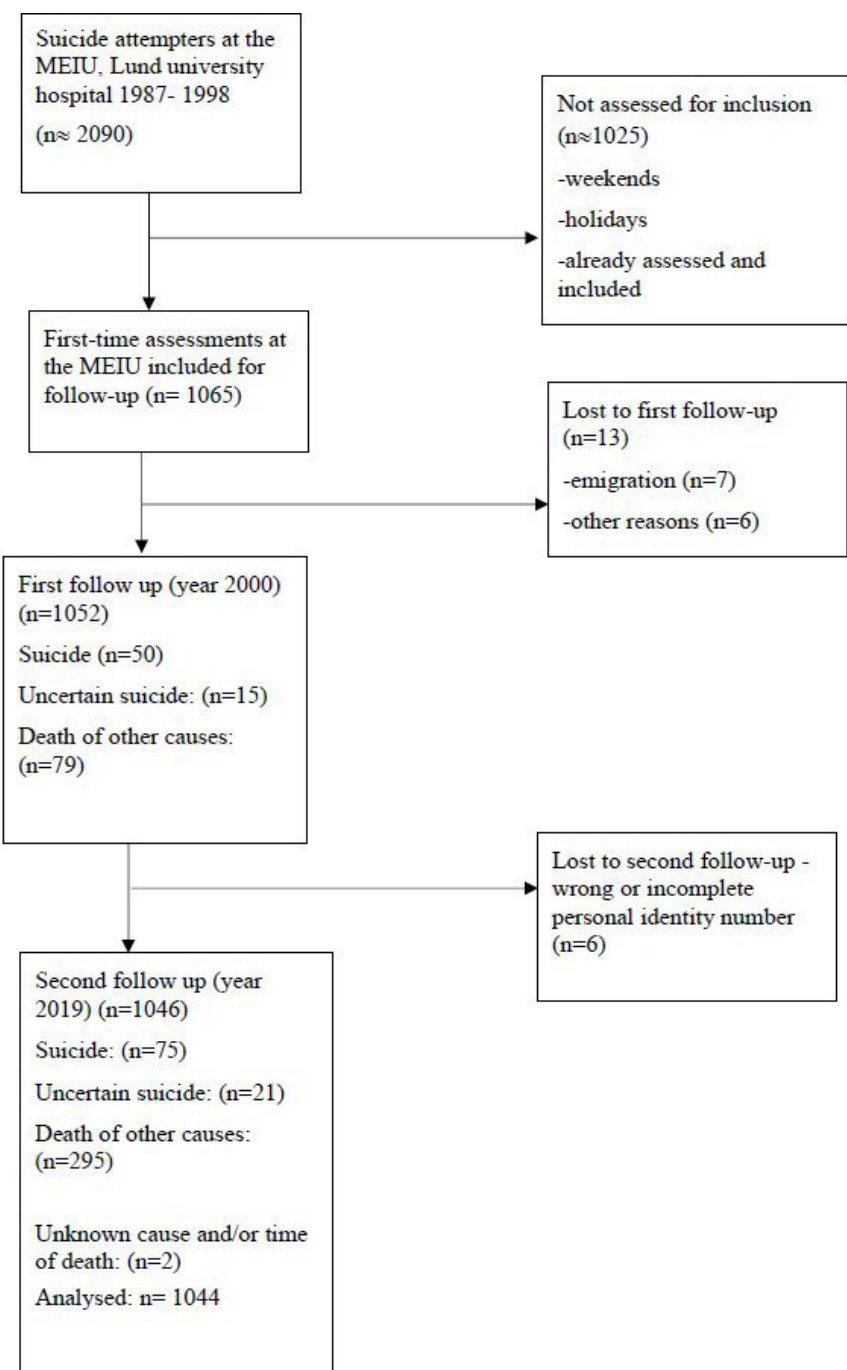

Figure 1 Flowchart of subjects through the study. MEIU, medical emergency inpatient unit.

by a psychiatrist and social worker and followed up after 21-32 years.

The sample was collected between 1987 and 1998 at a MEIU in southern Sweden, and represent approximately half of all individuals who were admitted to the MEIU following an attempted suicide during those years (estimated $1065 / 2000^{23}$ ). Individuals who were admitted on weekends or holidays were not assessed for inclusion, alongside individuals already included who reattempted (see figure 1). On weekdays, everyone who attempted suicide was assessed in a standardised manner with specific assessment measures (for more detailed description, $\left.\operatorname{se}^{28}{ }^{29}\right)$. Since most of the individuals were admitted on weekdays, a subsample of consecutive cases admitted also on weekends $(n=251)$ was compared with the rest of the sample. No statistically significant differences were found between the two groups for the frequency of suicide and overall mortality in either the previous follow-up study ${ }^{28}$ or in this sample.

Information about whether the study participant had died (date of death) or emigrated from Sweden (date of 
emigration) was obtained from the Swedish Tax until July 2019. Information about the causes of death was obtained from the Swedish Cause of Death Register, National Board of Health, the information in the register covered the time from the study start in January 1987 until 31 December 2018.

The previous follow-up by Skogman $e t a l^{28}$ identified male gender, age of 50 years or more at the time of index assessment, being a repeater, violent index method, total Suicide Intent Scale (SIS) score and major depression to be important risk factors for suicide. Furthermore, major depression was found a risk factor for both genders while violent method was a risk factor for men only, and older age as well as suicidal intention for women only.

We intended to include the 1052 individuals from the previous follow-up study. ${ }^{28}$ However, 8 individuals were lost to a second follow-up (2019), leaving 1044 participants for the present analyses (see figure 1). The mean observation time was 20 years (range $=0-32$ years) and the median was 22 years and 10 months. The total observation time was 20926 person-years. The mean age at index attempt was 40 years (range $=15-96$ years). At baseline, $42 \%$ were married or living with a partner and $55 \%$ were employed or studying. At discharge from the MEIU, 57\% were referred to an inpatient psychiatric ward. Frequency of risk factors in the total sample and in suicide are presented in table 1. In line with the previous follow-up of this population, ${ }^{28}$ we chose not to include uncertain suicides in the analysis of risk factors, due to the uncertain nature of these deaths.

The Strengthening the Reporting of Observational Studies in Epidemiology cohort reporting guidelines was used. $^{30}$

\section{Baseline investigation}

Baseline data were collected at emergency psychiatric consultations, that are always requested by the MEIU of patients who have attempted suicide. During the years 1987-1998, a semistructured interview that had been developed at the Suicide Research Centre based in the Department of Psychiatry at Lund University was in use. ${ }^{29}$ The assessment protocol was introduced to improve the procedure for treatment referral by ensuring a broad and standardised manner of assessment, including sociodemographic data, method of the suicide attempt, occurrence of previous attempts, suicidal intent and so on. The

Table 1 Risk factors for suicide after 0-32 years - whole sample analysis (Cox regression)

\begin{tabular}{|c|c|c|c|c|c|}
\hline Baseline variable & $\begin{array}{l}\text { Total sample \% (n)/ } \\
\text { median (IQR)* }\end{array}$ & $\begin{array}{l}\text { Suicide \% (n)/ } \\
\text { median (IQR)† }\end{array}$ & UHR (CI 95\%) & AHR (Cl 95\%) & $\begin{array}{l}\text { P value } \\
\text { (AHR) }\end{array}$ \\
\hline Sex & $n=1044$ & $n=75$ & & & \\
\hline Women (reference) & $61(633)$ & $53(40)$ & & & \\
\hline Age & $37(23)$ & $40(22)$ & & & \\
\hline Repeater & $43(415)$ & $62(42)$ & $2.24(1.37-3.65)$ & $1.62(0.97-2.70)$ & 0.068 \\
\hline Non-repeater (reference) & $57(555)$ & $38(26)$ & & & \\
\hline Suicide attempt method & $n=1044$ & & & & \\
\hline Violent method & $5(55)$ & $15(11)$ & 3.78 (1.99-7.17) & $2.53(1.26-5.09)$ & 0.009 \\
\hline Major depression & $16(171)$ & $33(25)$ & $5.15(2.54-10.48)$ & 2.63 (1.18-5.85) & 0.018 \\
\hline Dysthymia & $5(47)$ & $9(7)$ & $5.35(2.07-13.79)$ & $4.09(1.52-10.99)$ & 0.005 \\
\hline Psychosis & $7(69)$ & $16(12)$ & 5.61 (2.48-12.72) & 4.18 (1.73-10.09) & 0.001 \\
\hline Other & $41(427)$ & $27(20)$ & $1.50(0.72-3.13)$ & $1.12(0.49-2.54)$ & 0.795 \\
\hline \multirow[t]{2}{*}{ SIS score } & $\mathrm{n}=957$ & & & & \\
\hline & $13(12)$ & $18(9)$ & 1.09 (1.05-1.12) & $1.06(1.02-1.10)$ & 0.004 \\
\hline
\end{tabular}

The boldfaced entries mark statistically significant results.

*Percentage of the total sample of each category within the variable and number of individuals within each category, median and IQR are presented for the continuous variables age and SIS score.

†Percentage and number of all individuals who died by suicide within the category of the variable, and median and IQR are presented for the continuous variables age and SIS score.

AHR, adjusted HR; SIS, Suicide Intent Scale; UHR, unadjusted HR. 
assessments were carried out by a psychiatrist and a social worker. The psychiatrist diagnosed psychiatric disorders in accordance with Third Revised Edition of the Diagnostic and Statistical Manual for Mental Disorders (DSMIII-R) by clinical interviewing, but no structured interview was carried out for diagnostics. The evaluations were carried out in the emergency setting of the MIEU usually within 24 hours of the suicide attempt. Baseline in this study refers to the time of assessment at MEIU after the index suicide attempt.

\section{Variables}

With the exception of suicide, uncertain suicide and other causes of mortality, all other variables were assessed at the time of admission to the MEIU (baseline).

Suicide was defined as classified in the Cause of Death Register $^{31}$; external cause of morbidity and mortality; intentional self-harm in accordance to ICD- $10,{ }^{32}$ codes X60-X84 and ICD-9 ${ }^{33}$ codes starting with E95. Uncertain suicide was defined as classified in the Cause of Death Register $^{31}$; external cause of morbidity and mortality; event of undetermined intent in accordance to ICD- $10^{32}$ codes Y10-Y34 and ICD-9 ${ }^{33}$ codes starting with E98. Suicide attempt was defined as a situation in which a person has performed an actually or seemingly life-threatening behaviour with the intent of jeopardising their life or give the appearance of such an intent, but which has not resulted in death. ${ }^{34}$ Index suicide attempt refers to the suicide attempt that led to inclusion in this study. Repeaters refer to individuals who had made at least one such suicide attempt before the index suicide attempt. Violent method refers to the method of attempted suicide; either a method other than drug overdose or single wrist-cut, or a combination of different methods. ${ }^{35}$

$\mathrm{SIS}^{36}$ is a 20-item, clinician-completed measure of the severity of suicidal intent of a suicide attempt. A total score is computed based on the sum of the 0-2 severity ratings for the first 15 items. ${ }^{36}$ Missing data at the item level for the SIS were low with 905 of 1044 (86.7\%) of participants providing full data. To be included in the analyses, participants had to be missing a maximum of 3 items from the SIS $(n=52)$. In such cases, missing items were replaced by the mean of that individual's responses to the SIS. Cases with more than 3 values $(n=87)$ were excluded from further analysis of this variable.

Age was analysed as a numerical variable but divided into decades to facilitate interpretation. Psychiatric disorders were diagnosed according to the DSM-III-R. ${ }^{37}$ Only the main diagnosis was used for the purposes of the study. Secondary diagnoses, if any, were not used in the analyses due to very high rate of missing data. Psychosis, dysthymia, major depression and adjustment disorder were analysed separately in the regression analyses. The category 'other' includes substance abuse disorder (11\%), depression not otherwise specified (9\%), anxiety disorder (2\%), other axis I-diagnosis (4\%), no diagnosis (3\%), axis II diagnosis (but not an axis I diagnosis) (1\%) and cases with missing data for diagnosis (12\%). Observation time refers to the time patients were followed from the index date (time of assessment at the MEIU) until death, emigration or the study end.

\section{Data analysis}

Cox regression models were used to identify associations between baseline variables and time to suicide. The recommendations of Vittinghof $e t a l^{24}$ of at least 5-9 outcome events per covariate were followed by focusing on the clinically relevant variables identified at the earlier follow-up of this population. ${ }^{28}$ With these statistical limits we excluded variables viewed as more descriptive of the healthcare system than of the study participants, such as previous or current psychiatric contact and referral to psychiatric inpatient treatment.

All diagnostic groups (see the Variables section) were investigated by Kaplan-Meier survival analysis to plot temporal patterns of suicide after a suicide attempt. Three diagnoses were identified as possible risk factors for completed suicide, that is, major depression, dysthymia and psychosis. These were selected for further analysis. Substance abuse disorder, depression not otherwise specified, anxiety disorder, other axis I diagnosis or axis II diagnosis only were not identified as individual risk factors and were not included in further analysis. Adjustment disorder, the most common diagnosis (32\%), was chosen as the reference group. The HR for each of the selected diagnoses was analysed in relation to adjustment disorder using Cox regression.

To assess whether the risk factors for suicide differ over time, an analysis of Schoenfeld residuals was first performed to test the assumption of proportional hazards for each covariate separately. Separate Cox regression models were carried out for the first 5 years after the index attempt and for the remainder of the study period ( $>5$ years after index attempt). This 5-year cut-off was chosen pragmatically because approximately half of the suicides occurred in each of the two time spans ( 40 suicides in the first 5 years, 35 in the remaining years). HRs were calculated for each variable independently to identify possible risk factors. As a second step, variables that were found to be independently statistically significant in a regression model were included, thereby adjusting for the effect of these variables. Results were considered statistically significant when $p<0.05$.

We performed three sets of sensitivity analyses. Each set consisted of unadjusted Cox regression analyses for all covariates in both the short-term and the long-term analysis, but instead of using 5 years as the cut-off between short term and long term, the cut-off values used for the three sets were 3, 7 and 10 years, respectively. Altering the cut-off values changed the number of suicides in the short-term and long-term analyses, and we expected that fewer suicides would increase $p$ values and vice versa, while the HRs should remain approximately the same. Overall, HRs in the models were similar to the results in the main models, and the $\mathrm{p}$ values were comparable and overall effected in the expected direction.

All analyses were performed using V.25 of SPSS. ${ }^{38}$ 
Survival curve (suicide)

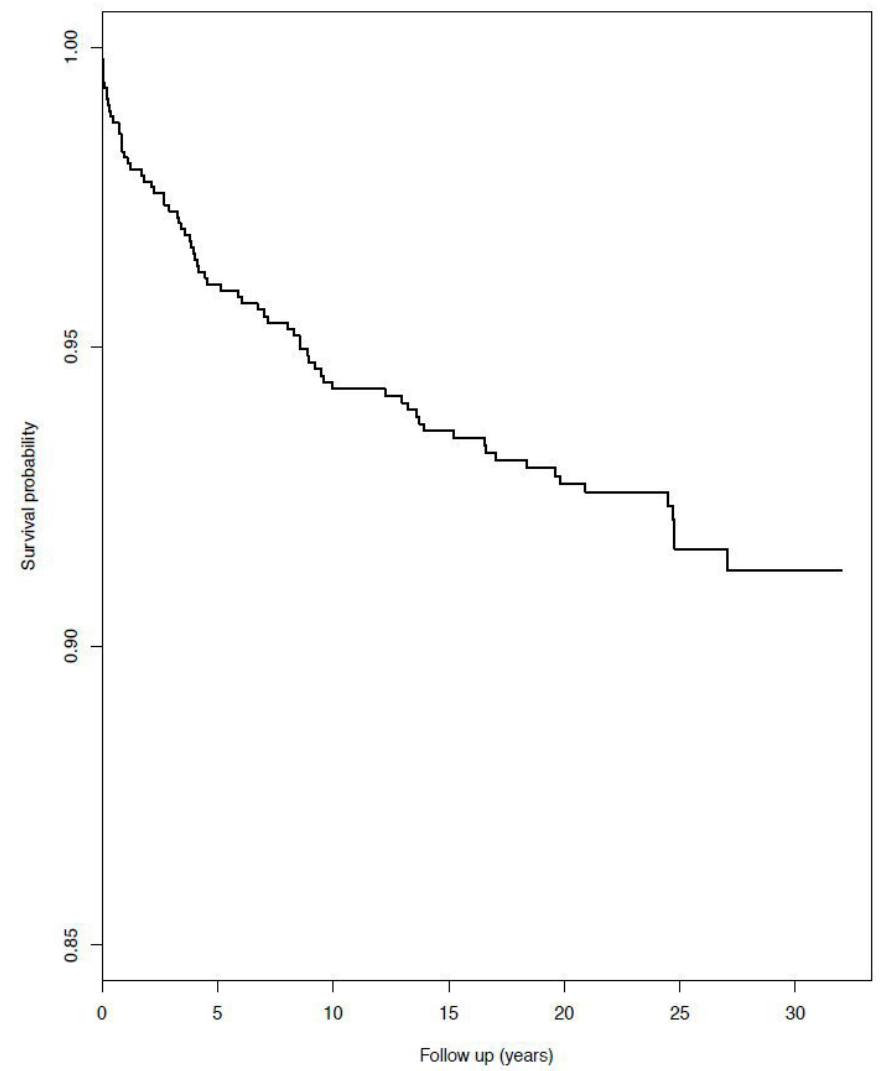

Figure 2 Survival curve of death by suicide in the study population.

\section{RESULTS}

\section{Suicide and all-cause mortality}

At follow-up, 393 individuals had died (37.6\%); 75 (7.2\%) by suicide, $21(2.0 \%)$ by deaths labelled as uncertain suicide, 295 (28.3\%) from other causes and two could not be analysed. The most common other cause of death was diseases of the circulatory system. The mean observation time in months was 88 (range=3-325 months) for those who died by suicide, 110 months for uncertain suicide (range=3-242 months) and 302 months for those who died of other causes (range $=0-384$ months).

As can be seen in figure 2, the incidence of suicide was higher in the first years after baseline. In the first year, 19 of the total 75 suicides occurred $(25.3 \%)$, and the following 2-5 years, another $21(28.0 \%)$. Within 10 years, another 16 deaths by suicide occurred $(21.3 \%)$. The remaining 19 suicides $(25.3 \%)$ were evenly distributed over the rest of the observation time. Regarding the overall mortality (391 individuals), 29\% died within 5 years of baseline. The mean age at death for all causes was 63 years (range $=22-100$ years, median $=64$ years) and for those who died by suicide, the mean age was 50 years (range $=22-81$ years, median $=48$ years $)$. There were equal proportions of men (47\%) and women (53\%) among those who committed suicide, while the proportions who died by uncertain suicide was higher among men $(71 \%)$ than women $(29 \%) \quad(\mathrm{p}<0.01)$.
Risk factors for suicide after 0-32 years: whole sample analysis

As can be seen in table 1 , the risk factors identified in the adjusted model for the total sample were violent method at index attempt, a diagnosis of major depression, dysthymia or psychosis, and the SIS score. Variables used to calculate the adjusted HR were age, occurrence of previous attempts, suicide attempt method, diagnosis and SIS score. For every increase of one point of the total SIS score the hazard for suicide increased by $6 \%$. Being diagnosed with psychosis at baseline was the risk factor with the highest HR. Age and being a repeater were found statistically significant as independent risk factors but no longer when included in the adjusted regression model. Analysis of the Schoenfeld residuals indicated a statistically significant violation of the assumption of proportional hazards for SIS score $(p=0.043)$, indicating that the HR for SIS was not consistent over the observation time. The Schoenfeld residual analysis was not significant for any of the other variables in the model, indicating that the proportional hazard assumption was not violated to a statistically significant degree.

\section{Risk factors for suicide at short term}

The significant risk factors for suicide at short term, that is, within 5 years of baseline, in the adjusted model, were a diagnosis of dysthymia and the SIS score (table 2). Variables used to calculate the adjusted HR were age, method of suicide attempt, diagnosis and the SIS score. The risk factor with the highest (adjusted) HR was being diagnosed with dysthymia. The SIS score was the most statistically significant predictor at short term. For every additional point increase on the SIS, the hazard for suicide increased by $10 \%$. Psychosis, major depression, age and violent method were statistically significant independent risk factors but no longer when included in the adjusted model.

\section{Risk factors at long term}

Long-term risk factors for suicide, that is, over 5 years after the index attempt (table 3), were being a repeater and having a diagnosis of major depression or psychosis. A diagnosis of psychosis at baseline, represented the risk factor with the highest (adjusted) HR at long-term follow-up, followed by major depression. Variables used to calculate the adjusted HR were occurrence of previous attempts, method of suicide attempt and diagnosis. The SIS score was not a statistically significant risk factor at long-term follow-up and was not included in the adjusted regression model.

\section{DISCUSSION}

Risk for suicide was found more than two decades after a suicide attempt that led to hospital admission in this large sample of Swedish adults. At follow-up, $7.2 \%$ of the individuals had died by suicide and of these, $53 \%$ had died within 5 years and $25 \%$ in the first year after the 
Table 2 Risk factors for suicide at short-term follow-up (Cox regression), $\mathrm{n}=1044$

\begin{tabular}{|c|c|c|c|}
\hline Baseline variable & UHR (CI 95\%) & AHR (Cl 95\%) & $\begin{array}{l}\text { P value } \\
\text { (AHR) }\end{array}$ \\
\hline Sex & $1.77(0.95-3.29)$ & - & - \\
\hline \multicolumn{4}{|c|}{ Reference: women $(n=1044)$} \\
\hline Age per 10 years $(n=1044)$ & $1.30(1.10-1.53)$ & $1.16(0.96-1.40)$ & 0.118 \\
\hline Repeater & $1.67(0.87-3.23)$ & - & - \\
\hline \multicolumn{4}{|c|}{ Reference: non-repeater ( $n=970)$} \\
\hline Violent method & $4.10(1.82-9.28)$ & $2.36(0.96-5.81)$ & 0.061 \\
\hline \multicolumn{4}{|c|}{ Reference: non-violent method ( $n=1044)$} \\
\hline \multicolumn{4}{|l|}{ Diagnosis } \\
\hline \multicolumn{4}{|c|}{ Reference: Adjustment disorder } \\
\hline Major depression & $5.43(2.12-13.87)$ & $2.32(0.85-6.35)$ & 0.101 \\
\hline Dysthymia & $6.72(2.05-22.02)$ & $5.09(1.51-17.10)$ & 0.009 \\
\hline Psychosis & $4.02(1.23-13.19)$ & $2.16(0.59-7.88)$ & 0.242 \\
\hline Other $(n=1044)$ & $1.04(0.36-3.00)$ & $0.58(0.18-1.93)$ & 0.377 \\
\hline SIS score $(n=957)$ & $1.13(1.07-1.20)$ & $1.11(1.04-1.17)$ & 0.001 \\
\hline
\end{tabular}

The boldfaced entries mark statistically significant results.

AHR, adjusted HR; SIS, Suicide Intent Scale; UHR, unadjusted HR.

index attempt. The risk factors at short-term and longterm follow-up differed. Risk factors for suicide at short term, that is, within 5 years, were violent method at index attempt, dysthymia and a higher SIS score. Analyses indicated that a higher SIS score was less relevant as a risk factor for suicides that occurred more than 5 years after the index attempt. The long-term risk factors for suicide, that is, over 5 years after the index attempt, were being a repeater and being diagnosed with major depression or psychosis. The presence of earlier suicide attempts before the index attempt proved to still be an important risk factor in a very long-term perspective.

This study has several strengths. A thorough, standardised assessment provided a broad set of clinical baseline data on a large clinical sample $(\mathrm{n}=1044)$ of suicide attempters. Furthermore, these were followed up for a very long time (mean $=20$ years, range $0-32$ years). To our knowledge, this is the largest population of suicide

Table 3 Risk factors for suicide at long-term follow-up (Cox regression), n=927

\begin{tabular}{|c|c|c|c|}
\hline Baseline variable & UHR (CI 95\%) & AHR (CI 95\%) & $\begin{array}{l}\text { P value } \\
\text { (AHR) }\end{array}$ \\
\hline Sex & $1.24(0.63-2.44)$ & - & - \\
\hline Age per 10 years $(n=927)$ & $1.16(0.92-1.50)$ & - & - \\
\hline Repeater & $3.18(1.50-6.71)$ & $2.68(1.24-5.78)$ & 0.012 \\
\hline \multicolumn{4}{|c|}{ Reference: non-repeater $(n=863)$} \\
\hline \multicolumn{4}{|c|}{ Reference: non-violent method $(n=927)$} \\
\hline \multicolumn{4}{|l|}{ Diagnosis } \\
\hline \multicolumn{4}{|c|}{ Reference: adjustment disorder } \\
\hline Major depression & $4.64(1.54-13.76)$ & $4.57(1.38-15.14)$ & 0.013 \\
\hline SIS score $(n=853)$ & $1.04(0.99-1.10)$ & - & - \\
\hline
\end{tabular}

The boldfaced entries mark statistically significant results.

AHR, adjusted HR; SIS, Suicide Intent Scale; UHR, unadjusted HR. 
attempters to be followed for this length of time with baseline data from an extensive clinical interview. This enabled statistical analysis of both short-term and longterm risk factors measured at baseline and comparisons of differences between these. Such comparisons have very rarely been done before, ${ }^{827}$ and never before with a follow-up time as long as in the present study. Nevertheless, generalisability to the wider population of individuals who attempt suicide is limited as this sample consists of individuals who were admitted to an MEIU as a consequence of attempted suicide, indicating a certain medical severity of the attempt.

Even though a standardised psychiatric assessment was carried out at the MEIU by a psychiatrist, the emergency context of the assessment can create difficulties in obtaining a full psychiatric history. This difficulty may help explain why no individuals in the present sample were diagnosed with bipolar disorder and quite few with personality disorders. It is likely that some of the patients among those diagnosed with major depression in fact suffered from bipolar depression. Indeed, it has been suggested that it is quite common in clinical practice that bipolar depression is misdiagnosed, often as major depression. ${ }^{39}$ Furthermore, suicide attempts in bipolar disorder often occur during depressive episodes. ${ }^{40}$ As only the main diagnoses could be used in the analysis, it is also possible that individuals could meet the criteria for other diagnoses than investigated in this study. Results must be interpreted in light of this limitation.

In comparison to the register-based population study by Tidemalm et $a l,{ }^{11}$ who specifically studied psychiatric diagnoses as risk factors for suicide, the present sample was much smaller and could not investigate as many diagnoses separately. However, diagnoses could be assessed in relation to other types of risk factors. As with most longitudinal studies, there were missing data for some variables, though efforts were made to statistically compensate for this in the case of the SIS. For statistical reasons, only a limited number of variables could be included in the regression models, thereby risking omission of potentially important variables, such as past psychiatric history and family history of mental illness. A limitation regarding the differential analysis in the shortterm and long-term subgroups was that the number of outcome events was further reduced, thereby diminishing statistical power to detect true associations. Finally, it is important to consider that there are some potentially important clinical variables that were not assessed at baseline and therefore not included in analysis. Also, some factors that were indeed assessed at baseline, would have been valuable to reassess during the follow-up period, for example, repeated attempts. ${ }^{9}$ Suicide risk is multifactorial, and complex interactions between different factors are probable. Furthermore, suicide risk is unlikely to be static over time and should be assessed at repeated intervals during an individual's lifetime.

The results of the present study that the risk of suicide after a suicide attempt persists at very long term confirm the results of four previous long-term follow-ups. ${ }^{10-13}$ The finding that $7.2 \%$ of the individuals had died by suicide is in line with the previous studies, similar to ours, that found that between $2 \%$ and $13 \%$ had died by suicide. ${ }^{10-13}$ Among these, a very early long-term follow-up of suicide attempters by Dahlgren published in $1977,{ }^{13}$ set in the same catchment area as the present study, found that $11 \%$ had died by suicide at follow-up after 35 years. Our finding that $53 \%$ of suicides occurred within 5 years and as much as $25 \%$ within the first year, is in agreement with earlier long-term follow-ups of suicide attempters that the risk of suicide persists for many years after a suicide attempt, though the incidence of suicide is highest within the first years. ${ }^{4-12}$

The results of the present study are to some extent in line with the findings of previous studies regarding mental disorder ${ }^{8274}$ and violent method. ${ }^{81421}$ SIS has been extensively used in research and high scores on the scale have repeatedly, but inconsistently, been linked to the risk of completed suicide ${ }^{5243}$ including the previous follow-up by Skogman $e t a t^{28}$ who found SIS score to be a relevant risk factor for women only at 6.5 years follow-up. A review from $2008^{42}$ found a positive association between high scores on SIS and suicide in 6 of 13 included studies, with follow-ups ranging from under 1 year to over 20 years. The present study found the SIS score highly statistically significant in the analysis of short-term risk factors but not in the long-term analysis, a finding which is also validated by the fact that the analysis revealed that the assumption of proportional hazards was violated for this variable. The finding that psychosis was the risk factor with the highest HR at long term is in line with previous findings that psychosis is a risk factor for suicide ${ }^{11}$ although its more evident importance at long term as compared with at short term has not previously been described. Furthermore, this study confirms the findings of previous studies that major depression is an important risk factor for suicide, which has been found both in the earlier follow-up of this population ${ }^{29}$ and in another sample of suicide attempters. ${ }^{11}$ Repeated suicide attempts have previously been identified as a risk factor for completed suicide at $2-9$ years, ${ }^{8} 4$ years ${ }^{11}$ and 10 years ${ }^{25}$ follow-up. The present study found that previous suicide attempts before baseline remained a risk factor even decades after the attempt.

The differences in short-term and long-term risk factors found in this study are to some extent in line with the findings of previous studies investigating such differences. ${ }^{8}{ }^{27}$ The finding of the present study that violent method seems a relevant short-term factor confirmed the results of Tidemalm $e t a l^{8}$ Maser $e t a l^{27}$ found symptoms of anxiety and affective states to be a relevant risk factor at short term (within 1 year). This is partially in line with our finding that the affective disorders (dysthymia and major depression) were relevant risk factors within the first 5 years, although we found that major depression continued to be a relevant risk factor at long term also. Furthermore, the present study identified suicidal intent 
as a relevant short-term risk factor. A possible explanation could be that suicidal intent, like affective and clinical symptoms of anxiety, may be more reflective of a state condition, the effect of which passes over time. On the other hand, when it comes to long-term factors these seem to be more trait-like and long-lasting, as impulsivity, which was found to be an important long-term risk factor (up to 14 years) by Maser $e t a l^{27}$ and, as found in the present study, that psychosis (often a chronic disorder) was a significant long-term risk factor.

\section{CONCLUSIONS}

The risk of suicide after a suicide attempt persists for up to 32 years after the index attempt. A baseline diagnosis of psychosis or major depression or earlier suicide attempts continued to be relevant risk factors at very long term, and the SIS score is a relevant risk factor within the first 5 years.

\section{Implications for clinicians and policymakers}

This study implies that individuals who have attempted suicide have a risk of suicide over several decades, with a particularly high risk within the first year after an attempted suicide. An earlier suicide attempt should be considered a risk factor for completed suicide regardless of when the attempt occurred. The severity of intent and the use of a violent method is important to consider at least the first 5 years following the attempt. It seems important to take on a very long-term-perhaps even a lifetime-perspective when it comes to secondary suicide preventive interventions for suicide attempters, especially those with repeated suicide attempts, and/or major depression and/or a psychotic disorder.

\section{Unanswered questions and future research}

Many possible risk factors, not included in this study, remain to be further investigated at long term, for example, life events after index attempt, heritability and so on. Future research would also benefit from follow-ups with assessment interviews of those still living.

Acknowledgements Professor emerita Lil Träskman-Bendz who was head of the Suicide Research Centre when the assessments at MEIU was established.

Contributors AÖ initiated the clinical baseline investigation and the present follow-up, acquired and registered the follow-up data and made revisions of the manuscript. JB initiated the follow-up, acquired the data, prepared the data set, designed the statistical strategy, made significant contributions to the statistical analysis and made revisions of the manuscript. AÖ, JB, SP-L and KSP contributed to designing the study and interpretation of the data. SP-L drafted the manuscript and made the statistical analysis. KSP made revisions of the manuscript. ÅW interpreted the data and made revisions of the manuscript. All authors also read and approved the final manuscript.

Funding This work was supported by the South Region Board, Region Skåne, National Board of Public Health (grant number N/A) and ALF-funding (grant number $N / A)$, Sweden.

Competing interests None declared.

Patient consent for publication Not required.

Ethics approval The study received approval from The Swedish Ethical Review Authority, no 2019-02602.

Provenance and peer review Not commissioned; externally peer reviewed.
Data availability statement Data are available upon reasonable request.

Open access This is an open access article distributed in accordance with the Creative Commons Attribution Non Commercial (CC BY-NC 4.0) license, which permits others to distribute, remix, adapt, build upon this work non-commercially, and license their derivative works on different terms, provided the original work is properly cited, appropriate credit is given, any changes made indicated, and the use is non-commercial. See: http://creativecommons.org/licenses/by-nc/4.0/.

ORCID iD

Sara Probert-Lindström http://orcid.org/0000-0003-0333-1887

\section{REFERENCES}

1 Fazel S, Runeson B. Suicide. N Engl J Med 2020;382:266-74.

2 Harris EC, Barraclough B. Suicide as an outcome for mental disorders. Br J Psychiatry 1997;170:205-28.

3 Hawton K, Zahl D, Weatherall R. Suicide following deliberate selfharm: long-term follow-up of patients who presented to a general Hospital. Br J Psychiatry 2003;182:537-42.

4 Tejedor MC, Díaz A, Castillón JJ, et al. Attempted suicide: repetition and survival--findings of a follow-up study. Acta Psychiatr Scand 1999;100:205-11.

5 Suominen K, Isometsä E, Ostamo A, et al. Level of suicidal intent predicts overall mortality and suicide after attempted suicide: a $12-$ year follow-up study. BMC Psychiatry 2004:4:11-17.

6 Suokas J, Suominen K, Lönnqvist J. Chronic alcohol problems among suicide attempters - post-mortem findings of a 14-year follow-up / Chronic alcohol problems among suicide attempters post-mortem findings of a 14-year follow-up. Nordic J Psychiatry 2005;1:45.

7 De Moore GM, Robertson AR. Suicide in the 18 years after deliberate self-harm a prospective study. Br J Psychiatry 1996;169:489-94.

8 Tidemalm D, Beckman K, Dahlin M, et al. Age-specific suicide mortality following non-fatal self-harm: national cohort study in Sweden. Psychol Med 2015;45:1699-707.

9 Wang AG, Mortensen G. Core features of repeated suicidal behaviour: a long-term follow-up after suicide attempts in a lowsuicide-incidence population. Soc Psychiatry Psychiatr Epidemiol 2006:41:103-7.

10 Jenkins GR, Hale R, Papanastassiou M, et al. Suicide rate 22 years after parasuicide: cohort study. BMJ 2002;325:1155.

11 Tidemalm D, Långström N, Lichtenstein P, et al. Risk of suicide after suicide attempt according to coexisting psychiatric disorder: Swedish cohort study with long term follow-up. BMJ 2008;337:a2205-31.

12 Suominen K, Isometsä E, Suokas J, et al. Completed suicide after a suicide attempt: a 37-year follow-up study. Am J Psychiatry 2004;161:562-3.

13 Dahlgren KG. Attempted suicides-35 years afterward. Suicide Life Threat Behav 1977;7:75-9.

14 Runeson B, Tidemalm D, Dahlin M, et al. Method of attempted suicide as predictor of subsequent successful suicide: national long term cohort study. BMJ 2010;341:c3222-c

15 Chen VCH, Tan HKL, Chen C-Y, et al. Mortality and suicide after self-harm: community cohort study in Taiwan. Br J Psychiatry 2011;198:31-6.

16 Suominen K, Isometsä E, Haukka J, et al. Substance use and male gender as risk factors for deaths and suicide--a 5-year follow-up study after deliberate self-harm. Soc Psychiatry Psychiatr Epidemiol 2004;39:720-4.

17 Nordström P, Samuelsson M, Asberg M. Survival analysis of suicide risk after attempted suicide. Acta Psychiatr Scand 1995;91:336-40.

18 Han B, Kott PS, Hughes A, et al. Estimating the rates of deaths by suicide among adults who attempt suicide in the United States. $J$ Psychiatr Res 2016:77:125-33.

19 Nielsen B, Wang AG, Brille-Brahe U. Attempted suicide in Denmark. IV. A five-year follow-up. Acta Psychiatr Scand 1990;81:250-4.

20 Cooper J, Kapur N, Webb R, et al. Suicide after deliberate self-harm: a 4-year cohort study. Am J Psychiatry 2005;162:297-303.

21 Christiansen E, Jensen BF. Risk of repetition of suicide attempt, suicide or all deaths after an episode of attempted suicide: a registerbased survival analysis. Aust N Z J Psychiatry 2007;41:257-65.

22 Runeson B, Haglund A, Lichtenstein P, et al. Suicide risk after nonfatal self-harm: a national cohort study, 2000-2008. J Clin Psychiatry 2016;77:240-6.

23 Niméus A, én M, Träskman-Bendz L. High suicidal intent scores indicate future suicide. Archive Suicide Res 2002;6:211-9. 
24 Vittinghoff E, McCulloch CE. Relaxing the rule of ten events per variable in logistic and COX regression. Am J Epidemiol 2007;165:710-8.

25 Nordentoft M, Breum L, Munck LK, et al. High mortality by natural and unnatural causes: a 10 year follow up study of patients admitted to a poisoning treatment centre after suicide attempts. BMJ 1993;306:1637-41.

26 Owens D, Wood C, Greenwood DC, et al. Mortality and suicide after non-fatal self-poisoning: 16-year outcome study. Br J Psychiatry 2005;187:470-5.

27 Maser JD, Akiskal HS, Schettler P, et al. Can temperament identify affectively ill patients who engage in lethal or near-lethal suicidal behavior? A 14-year prospective study. Suicide Life Threat Behav 2002;32:10-32.

28 Skogman K, Alsén M, Öjehagen A. Sex differences in risk factors for suicide after attempted suicide-a follow-up study of 1052 suicide attempters. Soc Psychiatry Psychiatr Epidemiol 2004;39:113-20.

29 Öjehagen A, Danielsson M, Träskman-Bendz L. Deliberate selfpoisoning: treatment follow-up of repeaters and nonrepeaters. Acta Psychiatr Scand 1992;85:370-5.

30 von Elm E, Altman DG, Egger M, et al. The strengthening the reporting of observational studies in epidemiology (STROBE) statement. Epidemiology 2007;18:800-4.

31 Socialstyrelsen. Cause of death register, 2018. Available: www. socialstyrelsen.se/statistik/statistikdatabas/dodsorsaker
32 WHO. International statistical classification of diseases and related health problems : ICD-10. 10th rev edn. World Health Organization, 1992.

33 WHO. International classification of diseases 9th revision, 1977.

34 Beck AT, Davis JH, Frederick CJ, et al. Classification and nomenclature, in suicide prevention in the seventies. Washington DC Government Printing Office, 1972.

35 Träskman L, Åsberg M, Bertilsson L, et al. Monoamine metabolites in CSF and suicidal behavior. Arch Gen Psychiatry 1981;38:631-6.

36 Beck AT, Herman I, Scuyler D. Development of suicidal intent scales. The predicion of suicide. Charles Press, 1974.

37 American Psychiatric Association. Diagnostic and statistical manual of mental disorders : DSM-III-R, 1987.

38 Norusis M. SPSS for windows. Chicago: SPSS Inc, 1995.

39 Bowden CL. Strategies to reduce misdiagnosis of bipolar depression. Psychiatr Serv 2001;52:51-5.

40 Valtonen HM, Suominen K, Mantere O, et al. Suicidal behaviour during different phases of bipolar disorder. J Affect Disord 2007;97:101-7.

41 Harris EC, Barraclough B. Suicide as an outcome for mental disorders. A meta-analysis. Br J Psychiatry 1997;170:205.

42 Freedenthal S. Assessing the wish to die: a 30-year review of the suicide intent scale. Arch Suicide Res 2008;12:277-98.

43 Stefansson J, Nordström P, Jokinen J. Suicide intent scale in the prediction of suicide. J Affect Disord 2012;136:167-71. 